\title{
A influência dos amigos no consumo de drogas entre adolescentes
}

\author{
Friend's influence on drug consumption \\ among adolescents
}

\author{
Luciana Roberta Donola CARDOSO ${ }^{1,2}$ \\ André MALBERGIER ${ }^{1,2}$
}

\begin{abstract}
Resumo
Objetivou-se avaliar a relação entre variáveis associadas aos amigos e o uso de álcool, tabaco e drogas ilícitas em 965 adolescentes de 50 escolas públicas de Jacareí e Diadema, São Paulo, Brasil. Foi utilizado o Drug Use Screening Inventory para a coleta de dados. Ter amigos que usam substâncias, que têm problemas com a lei, que roubaram/danificaram algo, que vendem drogas, que levam drogas a festas e que ficam entediados quando não bebem em festas aumentou progressivamente o risco de os adolescentes usarem álcool, tabaco e drogas ilícitas. Esse resultado mostra a importância de se avaliar o consumo dessas substâncias separadamente, já que o consumo concomitante de álcool e de tabaco parece ser mais grave do que o consumo isolado de um deles.
\end{abstract}

Palavras-chave: Abuso de álcool; Adolescente; Drogas ilícitas; Nicotina.

\begin{abstract}
This study aimed to evaluate the relationship between the variables associated with friends and alcohol, tobacco, and illicit drug use in 965 adolescents from 50 public schools in Jacarei and Diadema, São Paulo, Brazil. The Drug Use Screening Inventory was used for data collection. Having friends who use substances, who are in trouble with the law, who have stolen/damaged something, who sell drugs, who take drugs to parties, and who get bored when they do not drink at parties progressively increased the risk of adolescents using alcohol, tobacco, both of them, and illicit drugs. This result shows the importance of evaluating the consumption of these substances separately, as the consumption of alcohol and tobacco together seems to be more hazardous than consuming them individually.
\end{abstract}

Keywords: Alcohol abuse; Adolescent; Illicit drugs; Nicotine.

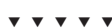

1 Universidade de São Paulo, Faculdade de Medicina, Departamento de Psiquiatria. São Paulo, SP, Brasil.

2 Universidade de São Paulo, Hospital das Clínicas, Grupo Interdisciplinar de Estudos de Álcool e Drogas. R. Dr. Ovídio Pires de Campos, 785, 05403-010, São Paulo, SP, Brasil. Correspondência para/Correspondence to: A. MALBERGIER. E-mail: <andre.malbergier@hcnet.usp.br>.

Agradecimentos: Organização das Nações Unidas para a Educação, Ciência e Cultura, Secretaria Estadual da Educação e Departamento do Governo do Estado de São Paulo.

Apoio: Secretaria Nacional de Políticas Antidrogas, Gabinete de Segurança Institucional da Presidência da República Federativa do Brasil (Processo no 004/2006). 
O consumo de substâncias psicoativas é um grave problema de saúde pública. O início do consumo de álcool e de outras drogas geralmente ocorre na adolescência e tem sido cada vez mais frequente nessa faixa etária. De acordo com o último Levantamento Nacional sobre o Consumo de Drogas Psicoativas, entre estudantes do Ensino Fundamental e Médio, 65,2\% fizeram uso de álcool, $24,9 \%$ de tabaco e $15,5 \%$ de maconha alguma vez na vida. Dentre eles, $11,7 \%$ relataram ter usado álcool, 3,8\% tabaco e 0,7\% maconha no mês anterior à entrevista (Centro Brasileiro de Informações Sobre Drogas Psicotrópicas, 2004).

Muitos fatores têm sido associados ao consumo de álcool e de drogas entre os adolescentes. Os amigos parecem exercer grande influência no início e na progressão do uso de álcool, de tabaco e de maconha entre adolescentes ( $D^{\prime}$ Amico \& McCarthy, 2006; Di Napoli, 2009; Ellickson, Tucker, Klein, \& Saner, 2004; Martino, Ellickson, \& McCaffrey, 2009). Fatores como o uso de álcool e de outras drogas, envolvimento em atividades ilegais, defasagem escolar, agressividade, rebeldia e comportamentos antissociais praticados pelos amigos têm sido associados ao uso de álcool e de outras drogas nessa fase da vida (D'Amico \& McCarthy, 2006; Duan, Chou, Andreeva, \& Pentz, 2009; Heavyrunner-Rioux \& Hollist, 2010).

De acordo com a literatura, os adolescentes são mais suscetíveis à opinião e à avaliação dos amigos (Duan et al., 2009; Heavyrunner-Rioux \& Hollist, 2010; Steinberg \& Monahan, 2007). A aprovação dos amigos é um fator que influencia muito o modo como o adolescente se comporta, o que aumenta o risco do uso de substâncias psicoativas. Muitas vezes, os amigos transmitem mensagem de supervalorização do uso de álcool, de tabaco e de outras drogas, de modo que o consumo promove popularidade no grupo. Além da valorização social, os adolescentes, muitas vezes, têm dificuldade para impor sua opinião, não resistindo à influência dos amigos, o que contribui para o uso de álcool e de outras drogas (Duan et al., 2009).

Estudo longitudinal com 4138 adolescentes entre 11 e 17 anos mostrou que ter amigos que 66 fumam e ter amigos que já experimentaram drogas aumentou em 3 vezes o risco de os adolescentes usarem tabaco (Ennett et al., 2008). Outro estudo mostrou que os adolescentes que relatam ter $50 \%$ de amigos fumantes e aqueles cujos melhores amigos fumam tiveram 2 vezes mais chances de fumar (Alexander, Piazza, Mekos, \& Valente, 2001).

Apesar de esse tema já ter sido discutido em publicações anteriores, até onde foi observado, nenhum estudo latino-americano avaliou a relação entre o consumo de substâncias psicoativas separadamente (apenas álcool, apenas tabaco, álcool e tabaco e drogas ilícitas - anfetamina, ecstasy, cocaína, crack, maconha, alucinógenos, tranquilizantes, ansiolíticos, esteroides, inalantes e solventes) e aspectos relacionados aos amigos.

O objetivo deste estudo foi verificar a relação entre variáveis associadas aos amigos e o uso de álcool, de tabaco, de ambos e de drogas ilícitas (separadamente) entre adolescentes de 10 a 18 anos.

\section{Método}

\section{Participantes}

A pesquisa foi realizada em 50 escolas públicas estaduais dos municípios de Jacareí e Diadema (SP) que ofereciam o Programa Escola da Família (PEF), uma iniciativa da Secretaria Estadual da Educação, com cooperação da Organização das Nações Unidas para a Educação, Ciência e Cultura (Unesco), que consiste em utilizar o espaço físico das escolas públicas estaduais, nos finais de semana, para desenvolver atividades socioeducativas com os jovens e suas famílias. Os adolescentes que utilizam esse programa não são, necessariamente, estudantes dessas escolas, mas, sim, moradores do bairro interessados em participar das atividades nos finais de semana.

Todos os adolescentes que participavam do PEF foram convidados a colaborar com a pesquisa. Os critérios de inclusão na pesquisa foram: ter idade entre 10 e 18 anos e participar desse programa nas escolas selecionadas. 


\section{Instrumento}

Utilizou-se o questionário Drug Use Screening Inventory (DUSI), já traduzido e validado no Brasil (De Micheli \& Formigoni, 2000), que avalia o uso de substâncias no mês anterior à entrevista e fatores relacionados ao seu uso.

O uso de álcool, de tabaco e de drogas ilícitas (anfetamina, ecstasy, cocaína, crack, maconha, alucinógenos, tranquilizantes, ansiolíticos, esteroides, inalantes e solventes) foi avaliado por uma questão na qual o adolescente assinalava o tipo e a frequência da droga que usou nos trinta dias anteriores à entrevista. As variáveis relacionadas aos amigos foram investigadas por meio de 16 questões referentes ao uso de álcool e outras drogas pelos amigos, comportamentos antissociais dos amigos, uso de álcool e de drogas em atividades de lazer e de diversão quando na companhia dos amigos, tempo que passam com os amigos, ter amigos e confiar neles.

As questões eram respondidas com "sim" ou "não": as respostas afirmativas equivalem à presença de problemas (De Micheli \& Formigoni, 2000). Para a aplicação desse instrumento, os autores treinaram cinquenta monitores do PEF.

O consumo de substância foi avaliado de acordo com cinco categorias: 1) não usou nenhuma substância; 2) usou apenas álcool; 3) usou apenas tabaco; 4) usou álcool e tabaco; e 5) usou drogas ilícitas. Na categoria álcool e tabaco, foram incluídos aqueles adolescentes que usaram as duas substâncias, mas não outras drogas. Na categoria drogas ilícitas, foram incluídos aqueles que usaram qualquer droga ilícita, podendo, também, ter usado álcool e/ou tabaco. O padrão do uso foi classificado como usou ou não usou. O critério utilizado foi baseado em estudos anteriores (De Micheli \& Formigoni, 2000; De Micheli, Fisberg, \& Formigoni, 2004). Foram realizadas análises univariadas com o teste Qui-quadrado, e análises múltiplas com teste de regressão logística binominal, e compararam-se o uso dessas substâncias separadamente e as variáveis relacionadas aos amigos. Consideraram-se como variável dependente o uso de álcool, de tabaco, de álcool e de tabaco e de drogas ilícitas separa- damente e como variáveis independentes das perguntas relacionadas aos amigos e das variáveis sociodemográficas. O método de seleção utilizado foi - Wald's Backward Test. A Oddes Ratio (OR) foi calculada com intervalo de confiança de $95 \%$ e nível de significância de 5\%.

Os dados descritos nos resultados e apresentados nas tabelas referem-se aos casos válidos, excluindo os sujeitos que não responderam à questão em estudo. Para as análises, utilizou-se o programa estatístico Statistical Package for the Social Sciences (SPSS), versão 15.0.

\section{Procedimentos}

O objetivo inicial desta pesquisa, realizada em 2007, era abranger mil adolescentes, 20 em cada escola. Entretanto, devido ao critério de inclusão e ao prazo estipulado para a coleta de dados, foi possível avaliar 965 adolescentes. Foram excluídos 6 adolescentes por terem menos de 10 ou mais de 18 anos.

Inicialmente, o projeto foi divulgado por meio de cartazes afixados na escola, que continham informações sobre a realização da pesquisa e sobre a participação dos interessados. Para estimular a participação dos adolescentes, os educadores falavam sobre a pesquisa durante as atividades programadas que aconteciam na escola. Os interessados informavam o nome e alguns dados residenciais ao monitor do programa e recebiam o termo de consentimento. $\mathrm{O}$ adolescente levava o termo ao pai ou responsável e o devolvia ao monitor antes da aplicação do questionário. Todos os adolescentes e seus responsáveis assinaram o termo de consentimento, o que garantiu a participação voluntária, sigilosa e o cumprimento das normas éticas de pesquisa com seres humanos. Após a assinatura do termo, os monitores agendavam com os adolescentes dia e horário para a aplicação do questionário. Os adolescentes recebiam o questionário, respondiam às perguntas (que eram dicotômicas: sim ou não) e o inseriam numa urna, sem precisar se identificar. Quando os adolescentes tinham alguma dúvida gramatical ou de compreensão das perguntas, os monitores podiam esclarecê-la. Entretanto, 
o monitor não poderia ler nenhuma pergunta do questionário. Antes de entregar o questionário, o monitor explicava ao adolescente o conteúdo das perguntas e a maneira como elas deveriam ser respondidas. Não houve nenhum questionamento por parte dos adolescentes durante o preenchimento dos questionários.

Todos os adolescentes que trouxeram o termo de consentimento assinado pelos pais ou responsáveis compareceram no dia indicado para responder ao questionário. Não houve recusa ou desistência dos adolescentes.

Este projeto foi aprovado pela Comissão de Ética do Hospital das Clínicas da Faculdade de Medicina da Universidade de São Paulo (Processo $n^{\circ}$ 425/06).

\section{Resultados}

A amostra foi constituída por 965 adolescentes, $436(45,2 \%)$ do sexo feminino e 529 $(54,8 \%)$ do sexo masculino; $831(86,1 \%)$ estavam no Ensino Fundamental, 132 (13,7\%) no Ensino Médio e $2(0,2 \%)$ não responderam a essa pergunta; $771(80,0 \%)$ nunca repetiram o ano escolar, $185(19,1 \%)$ repetiram o ano ao menos uma vez e $9(0,9 \%)$ não responderam; 606 (62,8\%) vivem com o pai e com a mãe, $218(22,6 \%)$ apenas com a mãe, $21(2,2 \%)$ apenas com o pai, $98(10,1 \%)$ com outros familiares, $2(0,2 \%)$ em instituição e 20 $(2,1 \%)$ não responderam. A idade média dos adolescentes foi de 13,5 (Desvio-Padrão=1,41) anos.

\section{Uso de substância entre os adolescentes}

Entre os 919 adolescentes que responderam às questões sobre consumo de drogas, $570(62,0 \%)$ não usaram nenhuma substância, 208 (22,6\%) usaram apenas álcool, 24 (2,6\%) usaram apenas tabaco, 54 (5,9\%) usaram álcool e tabaco e 63 (6,9\%) usaram alguma droga ilícita nos trinta dias anteriores à entrevista. As drogas ilícitas utilizadas foram: maconha $(n=27: 2,9 \%)$, tranquilizantes $(n=17$ :

$681,8 \%)$, anfetaminas $(n=15: 1,6 \%)$, ecstasy $(n=10$ :
$1,1 \%)$, inalantes $(n=10: 1,1 \%)$, cocaína $(n=8$ : $0,8 \%$, alucinógenos $(n=4: 0,4 \%)$ e anabolizantes $(n=4: 0,4 \%)$.

\section{Variáveis relacionadas aos amigos e uso de substâncias entre os adolescentes}

A Tabela 1 mostra os resultados obtidos nas análises univariadas. Nessas análises, foram comparadas as variáveis relacionadas aos amigos entre os adolescentes que fizeram uso de álcool, de tabaco, de ambos, e de drogas ilícitas, com aqueles adolescentes que não consumiram nenhuma substância.

Nessas análises, pôde-se observar que, em algumas variáveis relacionadas aos amigos, as razões de chance aumentam de acordo com a categoria da droga utilizada, de modo que o uso de álcool e de tabaco separadamente apresenta razões de chance menores do que o uso concomitante de álcool e de tabaco, que, por sua vez, mostra razões de chance menores do que o uso de drogas ilícitas. Os adolescentes que relataram ter amigos que usam regularmente álcool e/ou drogas tiveram 3,4 vezes mais chances de usar apenas álcool, 4,6 vezes mais chances de usar apenas tabaco, 7,2 vezes mais chances de usar álcool e tabaco e 8,6 vezes mais chances de usar drogas ilícitas do que aqueles que não tinham amigos que usavam drogas regularmente. Ter amigos que vendem ou dão drogas a outros jovens aumentou em 2,7 vezes o risco de os adolescentes usarem apenas álcool, em 5,8 vezes de usarem álcool e tabaco e 10,1 vezes o risco de usarem drogas ilícitas. Os adolescentes que relataram ter amigos que já tiveram problemas com a lei tiveram 2,1 vezes mais chances de usar apenas álcool, 4,9 vezes de usar apenas tabaco, 5,3 vezes mais chances de usar álcool e tabaco e 10,5 vezes mais chances de usar drogas ilícitas. Ter amigos que ficam entediados nas festas quando não é servido álcool aumentou em 2,4 vezes o risco de os adolescentes usarem apenas álcool, 3,5 vezes de usarem apenas tabaco, 3,8 vezes de usarem álcool e tabaco e 7,5 vezes o risco de usarem drogas ilícitas. Os adolescentes que relataram ter amigos que rouba- 
Tabela 1

Análise univariada da relação entre as variáveis associadas aos amigos e o uso de álcool, de tabaco, de ambos, e drogas ilícitas entre os adolescentes em comparação com aqueles que não usaram nenhuma substância. Jacarei e Diadema (SP)

\begin{tabular}{|c|c|c|c|c|c|c|c|c|}
\hline \multirow[t]{2}{*}{ Variáveis relacionadas aos amigos } & \multicolumn{2}{|c|}{$\begin{array}{l}\text { Usou apenas } \\
\text { álcool }\end{array}$} & \multicolumn{2}{|c|}{$\begin{array}{l}\text { Usou apenas } \\
\text { tabaco }\end{array}$} & \multicolumn{2}{|c|}{$\begin{array}{l}\text { Usou álcool e } \\
\text { tabaco }\end{array}$} & \multicolumn{2}{|c|}{$\begin{array}{l}\text { Usou drogas } \\
\text { ilícitas }\end{array}$} \\
\hline & $p$ & OR & $p$ & OR & $p$ & OR & $p$ & OR \\
\hline Ter amigos que usam álcool ou drogas regularmente & $<0,001$ & 3,4 & $<0,001$ & 4,6 & $<0,001$ & 7,2 & $<0,001$ & 8,6 \\
\hline Ter amigos que tiveram problemas com a lei & 0,002 & 2,1 & $<0,001$ & 4,9 & $<0,001$ & 5,3 & $<0,001$ & 10,5 \\
\hline A maioria dos seus amigos são mais velhos & 0,049 & 1,4 & 0,009 & 3,1 & 0,003 & 2,3 & $<0,001$ & 5 \\
\hline $\begin{array}{l}\text { Ter amigos que ficam entediados nas festas quando não é servi- } \\
\text { do álcool }\end{array}$ & $<0,001$ & 2,4 & 0,008 & 3,5 & $<0,001$ & 3,8 & $<0,001$ & 7,5 \\
\hline Ter amigos que levam drogas ou álcool às festas & $<0,001$ & 5 & 0,005 & 4,4 & $<0,001$ & 6,7 & $<0,001$ & 15 \\
\hline $\begin{array}{l}\text { Ter amigos que roubaram alguma coisa ou danificaram pro- } \\
\text { priedade de propósito }\end{array}$ & $<0,001$ & 2,3 & 0,003 & 4,3 & 0,001 & 3,4 & $<0,001$ & 8,6 \\
\hline Achar que os pais não gostam de seus amigos & - & - & 0,003 & 3,6 & $<0,001$ & 3,4 & $<0,001$ & 4,6 \\
\hline Sentir que não tem nenhum amigo em que possa confiar & - & - & - & - & 0,017 & 2,3 & 0,004 & 2,5 \\
\hline Preferir ter atividades com jovens bem mais velhos & - & - & 0,003 & 3,6 & - & - & $<0,001$ & 2,7 \\
\hline Ter amigo que vende ou dá drogas a outros jovens & $<0,001$ & 2,7 & - & - & $<0,001$ & 5,8 & $<0,001$ & 10,1 \\
\hline $\begin{array}{l}\text { Se sente infeliz com seu desempenho em atividades com os } \\
\text { amigos }\end{array}$ & 0,032 & 1,6 & - & - & 0,001 & 3 & - & - \\
\hline $\begin{array}{l}\text { Se sente incomodado por problemas que esteja tendo com os } \\
\text { amigos }\end{array}$ & 0,007 & 1,7 & - & - & - & - & 0,002 & 2,6 \\
\hline
\end{tabular}

Nota: OR: Oddes Ratio.

ram alguma coisa ou danificaram propriedade alheia tiveram até 4,3 vezes mais chances de usar apenas álcool, apenas tabaco e álcool e tabaco e 8,6 vezes mais chances de usar drogas ilícitas.

Ter amigos que levam drogas e/ou álcool a festas aumentou em até 5 vezes o risco de usar apenas álcool e apenas tabaco, 6,7 vezes o risco de usar álcool e tabaco e 15 vezes o risco de usar drogas ilícitas.

Os adolescentes que acham que os pais não gostam de seus amigos e aqueles que preferem ter amizade com jovens bem mais velhos tiveram entre 2,7 e 4,6 vezes mais chances de usar apenas tabaco, álcool e tabaco e drogas ilícitas do que aqueles que não usaram nenhuma substância.

Os adolescentes que relatam se sentir infelizes com seu desempenho nas atividades com os amigos tiveram 1,6 vez mais chances de usar álcool e 3 vezes mais chances de usar drogas ilícitas quando comparados aos que não se sentiam infelizes. Sentir-se incomodado por problemas com seus amigos aumentou em 1,7 vez o risco de usar álcool e 2,6 vezes o risco de usar drogas ilícitas. Sentir que não tem nenhum amigo em quem possa confiar aumentou em 2,3 vezes o risco de os adolescentes usarem álcool e tabaco e 2,5 vezes o risco de usarem drogas ilícitas.

A Tabela 2 mostra os resultados obtidos com a regressão logística. Os adolescentes que fizeram uso apenas de álcool tiveram entre 2,4 e 3,5 vezes mais chances de ter amigos que usam regularmente álcool e drogas e ter amigos que levam álcool e/ou drogas a festas. Os adolescentes que usaram apenas tabaco tiveram até 3,3 vezes mais chances de preferir ter amizade com jovens mais velhos e ter amigos que usam regularmente álcool e/ou drogas.

Entre os adolescentes que usaram álcool e tabaco, ter problemas de relacionamento com amigos devido ao seu uso de álcool e/ou de drogas foi o fator de maior associação (observado pela razão de chance $(\mathrm{OR}=8)$ ), seguido por ter amigos que usam álcool e/ou drogas regularmente $(\mathrm{OR}=4,6)$, sentir que não tem amigos em quem possa confiar $(\mathrm{OR}=2,5)$, ter amigos que vendem ou dão drogas a 
Tabela 2

Análise múltipla da relação entre variáveis relacionadas aos amigos e uso de álcool, de tabaco, de ambos, e de drogas ilícitas, em comparação com aqueles que não usaram nenhuma substância. Jacareí e Diadema (SP), 2007

\begin{tabular}{|c|c|c|c|c|}
\hline \multirow{2}{*}{$\begin{array}{l}\text { Variáveis } \\
\text { Usou apenas álcool }\end{array}$} & \multirow[t]{2}{*}{$p$} & \multirow[t]{2}{*}{ OR } & \multicolumn{2}{|c|}{ IC 95\% } \\
\hline & & & & \\
\hline Ter amigos que usam álcool/droga regularmente & 0,000 & 2,420 & 1,651 & 3,547 \\
\hline Ter amigos que levam álcool/drogas a festas & 0,000 & 3,510 & 2,095 & 5,881 \\
\hline Ter poucos amigos & 0,035 & 0,588 & 0,359 & 0,964 \\
\hline \multicolumn{5}{|l|}{ Usou apenas tabaco } \\
\hline Ter amigos que usam álcool/droga regularmente & 0,022 & 3,033 & 1,176 & 7,820 \\
\hline Preferir ter atividades com jovens mais velhos & 0,009 & 3,358 & 1,356 & 8,316 \\
\hline \multicolumn{5}{|l|}{ Usou álcool e tabaco } \\
\hline Ter amigos que usam álcool/droga regularmente & 0,000 & 4,637 & 2,155 & 9,975 \\
\hline Ter amigo que dá/vende drogas a outros jovens & 0,028 & 2,478 & 1,101 & 5,578 \\
\hline Achar que os pais não gostam de seus amigos & 0,030 & 2,242 & 1,081 & 4,647 \\
\hline Sentir que não tem amigos em quem possam confiar & 0,028 & 2,577 & 1,108 & 5,991 \\
\hline Ter problemas de relacionamento com amigos devido ao seu uso de álcool/drogas & 0,043 & 8,007 & 1,069 & 59,973 \\
\hline Ter poucos amigos & 0,031 & 0,347 & 0,132 & 0,910 \\
\hline Ensino Médio & 0,008 & 3,274 & 1,356 & 7,902 \\
\hline Ter repetido o ano escolar & 0,029 & 2,503 & 1,100 & 5,700 \\
\hline \multicolumn{5}{|l|}{ Usou drogas ilícitas } \\
\hline Ter amigos que tiveram problemas com a lei & 0,008 & 3,147 & 1,346 & 7,361 \\
\hline A maioria dos amigos são mais velhos & 0,003 & 3,257 & 1,479 & 7,171 \\
\hline Ter amigos que levam álcool/drogas a festas & 0,001 & 3,990 & 1,760 & 9,047 \\
\hline Ter problemas de relacionamento com amigos devido ao seu uso de álcool/drogas & 0,010 & 11,265 & 1,769 & 71,756 \\
\hline Ensino Médio & 0,018 & 3,0210 & 1,211 & 7,535 \\
\hline Ter repetido o ano escolar & 0,017 & 2,588 & 1,183 & 5,660 \\
\hline Não viver com o pai ou a mãe & 0,020 & 3,088 & 1,199 & 7,954 \\
\hline
\end{tabular}

Nota: OR: Oddes Ratio; IC: Intervalo de Confiança.

outros jovens $(O R=2,4)$ e achar que os pais não gostam de seus amigos $(\mathrm{OR}=2,2)$. Ter problemas de relacionamento com amigos devido ao seu uso de álcool e/ou drogas também foi o fator de maior associação entre os adolescentes que usaram drogas ilícitas $(O R=11,2)$, seguido por ter amigos que levam álcool e/ou drogas a festas $(\mathrm{OR}=3,9)$, a maioria dos amigos serem mais velhos $(O R=3,2)$ e ter amigos que tiveram problemas com a lei $(\mathrm{OR}=3,1)$. Ter poucos amigos diminuiu o risco de os adolescentes usarem apenas álcool e álcool e tabaco.

A regressão logística também mostrou que repetir o ano escolar foi associado ao uso de álcool e de tabaco e de drogas ilícitas entre os adolescentes. Os adolescentes do Ensino Médio tiveram 3,2 vezes mais chances de usar álcool e tabaco e 3 vezes mais chances de usar drogas ilícitas do que os adolescentes que não usaram nenhuma substân-

70 cia. Não viver com o pai e/ou com a mãe aumentou em 3 vezes o risco de os adolescentes usarem drogas ilícitas.

\section{Discussão}

Adolescentes mais velhos tiveram mais chances de usar álcool, tabaco e drogas ilícitas do que os mais novos, o que corrobora a literatura (Centro Brasileiro de Informações Sobre Drogas Psicotrópicas, 2004). Ter repetido o ano escolar também foi associado ao uso de álcool e tabaco e drogas ilícitas entre os adolescentes. Estudos nacionais mostraram que a repetência e a evasão escolar são mais frequentes em adolescentes que usam qualquer droga ilícita, mas não relacionam com o uso de álcool e tabaco (Bahls \& Ingbermann, 2005; Queiroz et al., 2001).

O uso de álcool, de tabaco e de drogas ilícitas foi associado às variáveis relacionadas aos amigos. 
Observou-se que a associação foi progressivamente maior (valor obtido pela razão de chance) para algumas variáveis relacionadas aos amigos de acordo com a categoria de sustâncias avaliadas. Adolescentes que usaram apenas álcool e apenas tabaco apresentaram níveis de associação menor do que os que usaram álcool e tabaco, que, por sua vez, apresentaram níveis de associação menor do que os que usaram drogas ilícitas.

Ter amigos que usam álcool ou drogas regularmente aumentou progressivamente a associação (observada pela razão de chance) para o uso de álcool, de tabaco, de ambos e de drogas ilícitas.

O uso de substâncias pelos amigos como fator de associação para o início e a progressão do uso de drogas tem sido mostrado em alguns estudos internacionais (Chen, Storr, \& Anthony, 2009; Degenhardt et al., 2009). Segundo D'Amico e McCarthy (2006), ter amigos que usam álcool foi um fator preditor para o uso de álcool e de maconha entre os adolescentes, e o uso de maconha pelos amigos aumentou o consumo de álcool entre os adolescentes. Essa relação tem sido explicada por meio de algumas teorias comportamentais, dentre elas a Teoria da Aprendizagem Social. De acordo com Bandura (1977), os indivíduos aprendem a se comportar ao observar o comportamento de seus pares. Consequências positivas decorrentes do comportamento, como valorização social, sensação de pertencimento ao grupo e popularidade, são responsáveis pela aprendizagem. Por exemplo, os jovens observam que os adolescentes que consomem álcool e outras drogas são mais populares e valorizados no grupo. Para obter essa consequência (valorização social), o jovem imita o comportamento daquele mais popular (o líder do grupo).

Estudo dinamarquês mostrou que os alunos que viam outros alunos e professores fumando nos recintos da escola apresentaram mais chances de fumar do que aqueles que não os viam fumando. Os autores sugerem que os ver fumar passava uma mensagem de permissividade e tolerância ao fumo, além de muitos desses serem vistos como referências para o aluno (Poulsen et al., 2002). O escalonamento do uso de álcool, de tabaco e de maconha também tem sido explicado por essa teoria quando uma das variáveis de controle é a influencia dos amigos (Bot, Engels, Knibbe, \& Meeus, 2007; Ennett et al., 2006). Nesses estudos, não só a observação do uso de álcool pelos amigos aumenta o risco de os adolescentes usarem drogas ilícitas, mas, também, a simples avaliação do que eles acham que os amigos fazem ou vão achar de seu comportamento os motiva a consumir substâncias psicoativas, o que aumenta o risco do uso.

A prática de comportamentos antissociais pelos amigos também foi evidenciada na literatura como um fator de risco para o uso de drogas pelos adolescentes (Martino, Collins, Ellickson, Schell, \& McCaffrey, 2006; Warr, 2005). Neste estudo, os adolescentes que relataram ter amigos com problemas com a lei, que roubaram ou danificaram algo e que vendem drogas tiveram progressivamente 0 risco do uso de álcool, de tabaco, de ambos e de drogas ilícitas aumentado. De acordo com alguns autores, usar droga já é considerado um comportamento transgressor, o que se enquadraria nos comportamentos antissociais (Epstein, Williams, Botvin, Diaz, \& III-Williams, 1999; Fuemmeler, Taylor, Metz Jr., \& Brown, 2002). Desse modo, esse comportamento pode novamente ser explicado pela Teoria da Aprendizagem Social. Os jovens que estão inseridos em ambientes nos quais transgredir regras é considerado "legal" apresentam maior risco de consumir substâncias psicoativas. Walker-Barnes e Mason (2004), em um estudo longitudinal com 300 estudantes do Ensino Médio, mostraram que o envolvimento com gangue era mais frequente entre os adolescentes que usaram álcool e drogas ilícitas. Recentemente, Swahn, Bossarte, West e Topalli (2010) observaram que os adolescentes que beberam mais de 3 doses no mês anterior à entrevista apresentaram quase 10 vezes mais chances de se envolver em gangue do que quem não bebeu. Nesse estudo, o uso de drogas ilícitas foi 6 vezes maior entre os que pertenciam a gangues.

O uso de álcool e de drogas entre adolescentes em situação social com os amigos também tem sido enfatizado nos estudos que avaliam os fatores de risco para o uso de substâncias entre os adolescentes (Hoffaman, Sussman, Unger, \& Valent, 
2006; Kliewer \& Murrelle, 2007). Ter amigos que levam drogas em festas e que ficam entediados caso não seja servido álcool em festas aumentou progressivamente a associação entre essas variáveis e o uso de álcool, tabaco, ambos, e drogas ilícitas. Os amigos são considerados a estrutura de lazer e contato social mais importante para os adolescentes. Falta de suporte e monitoramento familiar, dificuldade de relacionamento e comunicação com os pais podem favorecer o uso de álcool e de drogas em situação de lazer (Tobler \& Komro, 2010).

Diversos fatores relacionados aos amigos discutidos neste artigo têm sido apontados como indicadores de risco para o uso de substâncias entre os adolescentes. Todavia, até onde se observou, não há nenhum estudo latino-americano que mostre um aumento progressivo na associação entre consumo de álcool, de tabaco, de ambos, e de drogas ilícitas e as variáveis relacionadas aos amigos. Esse é o principal achado deste estudo. O consumo das duas drogas lícitas mais comuns parece ser mais grave do que o consumo de alguma delas isoladamente. A categoria "usuário de álcool e tabaco" separada da categoria daqueles que usaram apenas álcool ou apenas tabaco parece merecer maior destaque, já que os adolescentes que usaram as duas substâncias apresentaram maior associação do que aqueles que as consumiram separadamente.

Esses resultados podem ser um alerta para a progressão do uso de drogas entre os adolescentes. Mesmo não sendo um estudo longitudinal, as razões de chance encontradas nas análises mostram que, conforme os adolescentes apresentam maior número de situação de risco para uso de drogas com amigos, maiores as chances de apresentarem um padrão mais grave de consumo.

Neste estudo, observou-se que parece existir uma relação crescente entre categorias de consumo de substâncias e presença de variáveis de risco de uso de drogas associadas ao círculo social dos adolescentes. Esse resultado mostra a importância de se avaliar o consumo de substâncias de acordo com as categorias "apenas álcool", "apenas tabaco", "álcool e tabaco" e "drogas ilícitas", já que o consumo das duas drogas lícitas mais comuns parece ser mais grave, do ponto de vista social, do que o consumo de alguma delas isoladamente.

Este estudo, por sua transversalidade, não foi capaz de avaliar se as associações encontradas são causais nem a possível direção da causalidade. Tanto as variáveis relacionadas aos amigos podem aumentar as chances de os adolescentes consumirem álcool, tabaco e drogas ilícitas como o consumo dessas substâncias aumenta o risco de os adolescentes terem amigos que usam drogas, apresentam comportamentos antissociais e relacionam uso de drogas e de álcool ao lazer.

Vieses de amostragem (apenas 50 escolas em dois municípios do estado de São Paulo foram avaliadas) e de autosseleção (adolescentes tinham o controle sobre a possibilidade de participação) não podem ser descartados e podem comprometer a generalização dos resultados para toda a população brasileira. Entretanto, o tamanho da amostra (quase 1 mil adolescentes) é uma das virtudes deste estudo, que pode servir de estímulo para levantamentos nacionais sobre tema tão relevante.

\section{Referências}

Alexander, C., Piazza, M., Mekos, D., \& Valente, T. (2001). Peers, schools, and adolescent cigarette smoking. Journal of Adolescent Health, 29(1), 22-30.

Bahls, F. R. C., \& Ingbermann, Y. K. (2005). Desenvolvimento escolar e abuso de drogas na adolescência. Estudos de Psicologia (Campinas), 22(4), 395-402. doi: 10.1590/S0103-166X2005000400007

Bandura, A. (1977). Social learning theory. Englewood Cliffs, NJ: Prentice-Hall.

Bot, S. M., Engels, R. C., Knibbe, R. A., \& Meeus, W. H. (2007). Sociometric status and social drinking: Observations of modeling and persuasion in young adult peer groups. Journal of Abnormal Child Psychology, 35(6), 929-941.

Centro Brasileiro de Informações Sobre Drogas Psicotrópicas. (2004). V Levantamento nacional sobre o consumo de drogas psicotrópicas entre estudantes do ensino fundamental e médio da rede pública de ensino nas 27 capitais brasileiras. São Paulo: Unifesp. Recuperado em outubro 20, 2011, disponível em: <http:// www.unifesp.br/dpsicobio/cebrid/>.

Chen, C. Y., Storr, C. L., \& Anthony, J. C. (2009). Early-onset drug use and risk for drug dependence problems. Addictive Behaviors, 34(3), 319-322. 
D'Amico, E. J., \& McCarthy, D. M. (2006). Escalation and initiation of younger adolescents' substance use: The Impact of perceived peer use. Journal of Adolescent Health, 39(4), 481-487.

Degenhardt, L., Chiu, W. T., Conway, K., Dierker, L., Glantz, M., Kalaydjian, A., et al. (2009). Does the 'gateway' matter? Associations between the order of drug use initiation and the development of drug dependence in the National Comorbidity study replication. Psychological Medicine, 39(1), 157-167.

De Micheli, D., \& Formigoni, M. L. O. S. (2000). Screening of drug use in a teenage brazilian sample using the Drug Use Screening Inventory (DUSI). Addictive Behaviors, 25(5), 683-691.

De Micheli, D., Fisberg, M., \& Formigoni, M. L. (2004). Study on the effectiveness of brief intervention for alcohol and other drug use directed to adolescents in a primary health care unit. Revista da Associação Médica Brasileira, 50(3), 305-313.

Di Napoli, P. P. (2009). Early initiation of tobacco use in adolescent girls: Key sociostructural influences. Applied Nursing Research, 22(2), 126-132.

Duan, L., Chou, C. P., Andreeva, V. A., \& Pentz, M. A. (2009). Trajectories of peer social inuences as longterm predictors of drug use from early through late adolescence. Journal of Youth and Adolescence, 38(3), 454-465.

Ellickson, P. L., Tucker, J. S., Klein, D. J., \& Saner, H. (2004). Antecedents and outcomes of marijuana use initiation during adolescence. Preventive Medicine, 39(5), 976-984.

Ennett, S. T., Bauman, K. E., Hussong, A., Faris, R., Foshee, V. A., DuRant, R. H., et al. (2006). The peer context of adolescent substance use: Findings from social network analysis. Journal of Research on Adolescence, 16(2), 159-186.

Ennett, S. T., Faris, R., Hipp, J., Foshee, V. A., Bauman, K. E., Hussong, A. et al. (2008, July). Peer smoking, other peer attributes, and adolescent cigarette smoking: A social network analysis. Prevention Science, 9(2), 88-98.

Epstein, J. A., Williams, C., Botvin, G. J., Diaz, T., \& IIIWilliams, M. (1999). Psychosocial predictors of cigarette smoking among adolescents living in public housing developments. Tobacco Control, 8(1), 45-52.

Fuemmeler, B. F., Taylor, L. A., Metz Jr., A. E., \& Brown, R. T. (2002). Risk-taking and smoking tendency among primarily African American school children: Moderating inuences of peer susceptibility. Journal of Clinical Psychology in Medical Settings, 9(4), 323-330.

Heavyrunner-Rioux, A. R., \& Hollist, D. R. (2010). Community, family, and peer influences on alcohol, marijuana, and illicit drug use among a sample of native American youth: An analysis of predictive factors. Journal of Ethnicity in Substance Abuse, 9(4), 260-283.

Hoffaman, B. R., Sussman, S., Unger, J. B., \& Valente, T. $W$. (2006). Peer inuences on adolescent cigarette smoking: A theoretical review of the literature. Substance Use \& Misuse, 41(1), 103-155.

Kliewer, W., \& Murrelle, L. (2007). Risk and protective factors for adolescent substance use: Findings from a study in selected Central American countries. Journal of Adolescent Health, 40(5), 448-455.

Martino, S. C., Collins, R. L., Ellickson, P. L., Schell, T. L., \& McCaffrey, D. (2006). Socio-environmental influences on adolescents' alcohol outcome expectancies: A prospective analysis. Addiction, 101(7), 971-983.

Martino, S. C., Ellickson, P. L., \& McCaffrey, D. F. (2009). Multiple trajectories of peer and parental influence and their association with the development of adolescent heavy drinking. Addictive Behaviors, 34(8), 693-700.

Poulsen, L. H., Osler, M., Roberts, C., Due, P., Damsgaard, M. T., \& Holstein, B. E. (2002). Exposure to teachers smoking and adolescent smoking behaviour: Analysis of cross sectional data from Denmark. Tobacco Control, 11(3), 246-251.

Queiroz, S., Scivoletto, S., Silva, M. M. S., Strassman, P., Andrade, A. G., \& Gattaz, W. F. (2001). Uso de drogas entre estudantes de uma escola pública de São Paulo. Revista de Psiquiatria Clínica, 28(4), 176-182.

Steinberg, L., \& Monahan, K. C. (2007). Age differences in resistance to peer influence. Developmental Psychology, 43(6), 1531-1543.

Swahn, M. H., Bossarte, R. M., West, B., \& Topalli, V. (2010). Alcohol and drug use among gang members: Experiences of adolescents who attend school. Journal of School Health, 80(7), 353-360.

Tobler, A. L., \& Komro, K. A. (2010). Trajectories or parental monitoring and communication and effects on drug use among urban young adolescents. Journal of Adolescent Health, 46(6), 560-568.

Walker-Barnes, C. J., \& Mason, C. A. (2004). Delinquency and substance use among gang-involved youth: The moderating role of parenting practices. American Journal of Community Psychology, 34(3-4), 235-250.

Warr, M. (2005). Making delinquent friends: Adult supervision and children's affiliations. Criminology, 43(5), 77-106.

Recebido em: 1/3/2012

Versão Final em: 13/9/2012

Aprovado em: 3/10/2012 
